\title{
Design and Research of Remote Monitoring Terminal of Tomato Harvester Based on BDS and GPRS
}

\author{
Bin $\mathrm{Li}^{1}$, Danyang $\mathrm{Li}^{2}$ and Jiangquan $\mathrm{Li}^{1 *}$ \\ Machinery and Electrical Engineering College, Shihezi University \\ Xinjiang Shihezi, 832000 \\ *ljq6949@163.com
}

\begin{abstract}
To meet the requirements of tomato harvesting enterprises of scheduling tomato harvesting machine reasonably and real-time monitoring of field operations during the harvest in the autumn, this article designed remote monitoring terminal based on Bei Dou Navigation Satellite System (BDS) and General Packet Radio Service (GPRS), which formed remote monitoring system with communication system and monitoring center. The running states and position information of tomato harvesters were transmitted to the monitoring center by the monitoring terminal via the GPRS and Internet, and then stored and analyzed by monitoring center. The design ensured the monitoring center that could realize several functions, such as real-time display of position of tomato harvester and historical track of harvesting operation. In addition, the remote monitor terminal could provide technical supports of safety production and scientific control for tomato harvesting enterprises and would be significant to the informatization of tomato harvesting management.
\end{abstract}

Keywords: Tomato Harvester; BDS; GPRS; Remote Monitoring

\section{Introduction}

Focusing on the urgent needs of tomato harvester in security, operations management, emergency maintenance [1], and other aspects in large-scale mechanized harvesting tomatoes area, this paper designs remote monitoring terminal for tomato harvester. As an important part of remote monitoring system [2-3], remote monitoring terminal could implement remote monitoring of tomato harvester and scientific management of enterprises, and ensure the tomato harvesting enterprises scheduling tomato harvester reasonably to improve the operation efficiency and economic benefits.

\section{Compositions of Monitoring System of the Tomato Harvester}

Remote monitoring system of tomato harvester was mainly composed of monitoring terminal, mobile operator of network, the Internet and the monitoring center [4-5], as shown in Figure 1.

1) Monitoring terminal

Monitoring terminal has functions of collecting tomato harvester operation data, positioning information, communicating via GPRS, and receiving and setting up SMS commands such as IP address and port number.

2) Mobile operator network and the Internet

Owning to the domestic mobile operators network has basically realized full-area covering, GPRS communication mode has advantages of low cost [6], high reliability and

${ }^{*}$ Corresponding Author 
satisfying the demands of remote monitoring of tomato harvester in harvesting area. Therefore, the monitoring terminal adopted mobile operator network and the Internet as medium for data communication.

3) Monitoring center

The monitoring center has functions of receiving, analyzing, and storing the data uploaded by monitoring terminal in tomato harvester, and finally displays the data on the electronic map [7]. According to the data uploaded, the position and operation state of the tomato harvester could be determined, which in turn could fulfill the remote real-time monitoring, information management and statistical analysis.

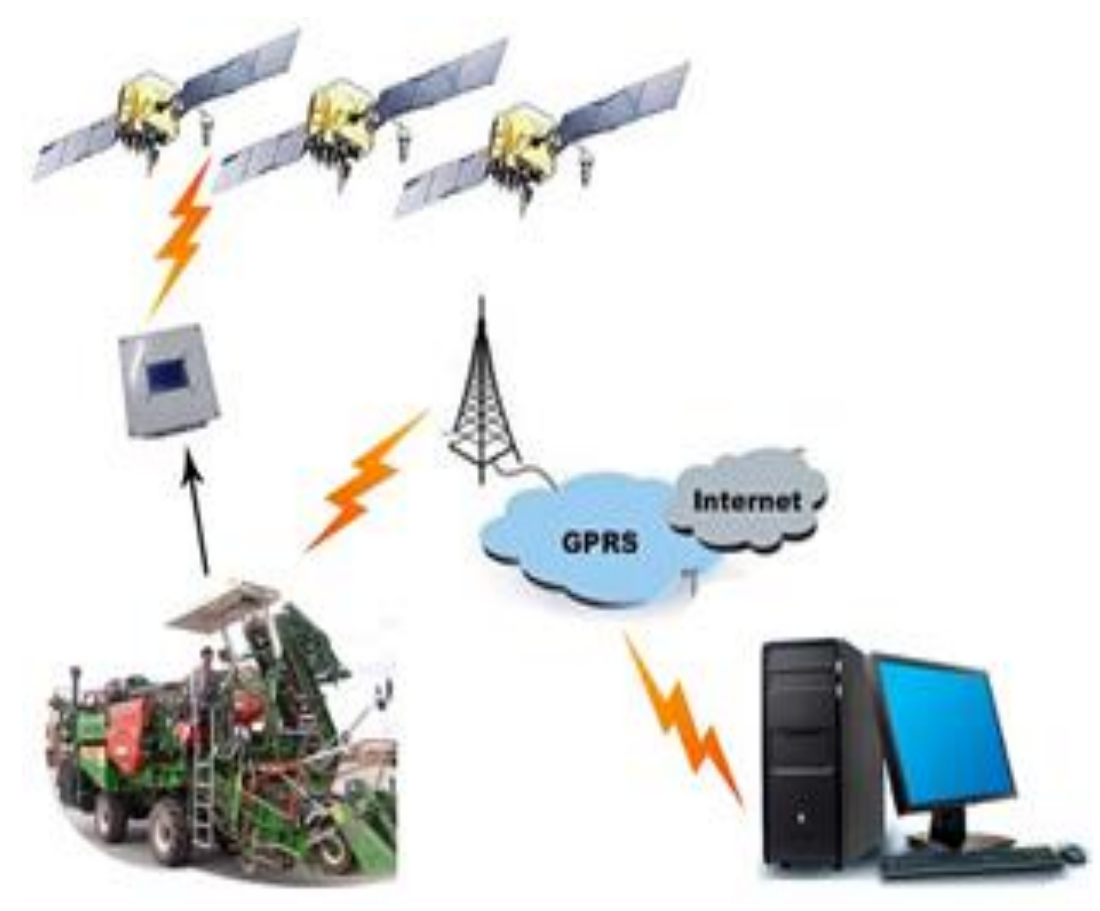

Figure 1. Remote Monitoring System of Tomato Harvester

\section{Hardware Design of Remote Monitoring Terminal}

\subsection{Hardware Structure}

Remote monitoring terminal adopted modular design, which included the power supply module, data acquisition module, positioning module, communication module and micro control unit module (MCU), etc. Since the operating voltage in each part of the terminal was different, the design of the power supply circuit module was multi-output circuit interface. Data acquisition module was mainly composed of vehicle status display, digital signal and analog signal acquisition interface circuit. Positioning module was applied to position the tomato harvester. Communication module was utilized for data communication. All modules were connected with the MCU module, which constituted the whole hardware system. The hardware structure of monitor terminal is shown in Figure 2. 


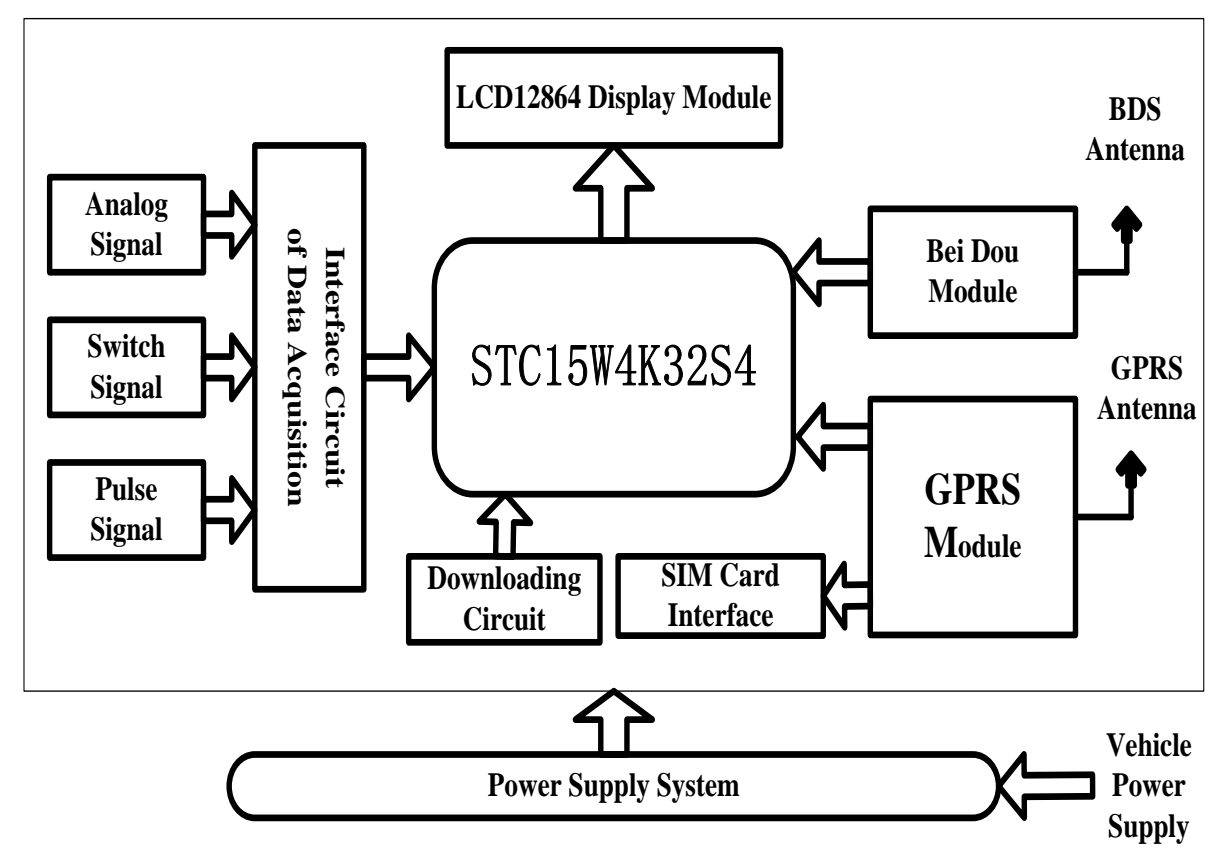

Figure 2. The Hardware Structure of Monitor Terminal

\subsection{Design of Hardware Circuit}

\subsubsection{Type Selection of the Main Module}

\section{1) MCU module}

MCU used to achieve exchanges and treatments of information was the core of the monitoring terminal. This design chose STC15W4K32S4 (SysTem Chip) as the MCU module with one-clock period per machine cycle (1T) enhanced 51 single chip microcomputer. Its internal integrates high reliability reset circuit, high precision crystal oscillator circuit with operating frequency up to $28 \mathrm{MHz}, 32 \mathrm{k}$ FLASH program memory, $4 \mathrm{k}$ SRAM, four UART, five timers, 8 high-speed 10 bit A/D conversion interfaces and up to 62 fast I/O ports. The MCU module could meet the needs of the terminal system in speed, memory space, serial communication, and signal acquisition. The range of its operating temperature is $-40 \sim 85^{\circ} \mathrm{C}$, which would be suitable to outdoor working environment of the tomato harvester.

\section{2) BDS module}

With the rapid development of Bei Dou satellite navigation system and the quick upgrading of positioning module technology in recent years, BDS module has possessed the functions of global positioning system (GPS) and "Bei Dou" dual mode navigation. In this design, the BDS module was used as positioning module of the monitoring terminal considering its better positioning precision and stable performance compared with the only GPS module.

BDS module was introduced to locate the operation position of the tomato harvester. UM220 module (Unicore communications Inc) was chosen in this design. This module had the properties of low power consumption, small volume, built into the metal shell and high reliability, which made it could be used in the working environment of the monitoring terminal system.

\section{3) GPRS Module}

GPRS module was applied to implement the communication between monitoring terminal and monitoring center. With the good performances of compact appearance, 
stable, cost-effective, and TCP/IP protocol stack inside, the design used Huawei GTM900-C compact module which could satisfy multiple demands of users.

\subsubsection{Main Circuit Design}

\section{1) Data Acquisition Module}

Signals collected by the monitor terminal consisted of pulse signal, switch signal and analog signal. Since the pulse signal frequencies produced by the proximity switch in the detection of engine, blower and transmission gear were low, the design applied the processing circuit which has the same signal with switch signal. Analog acquisition circuit converted the vehicle analog signals such as oil temperature and diesel mass of the tomato harvester to standard voltage signals. The acquisition circuit of pulse signal and switch signal is illustrated in Figure 3.

In order to facilitate tractor driver checking terminal operation information like the location, date, time, GPRS connection state, engine speed, harvesting area, and alarm status, the module added liquid crystal display LCD12864 and state indicator interface circuits.
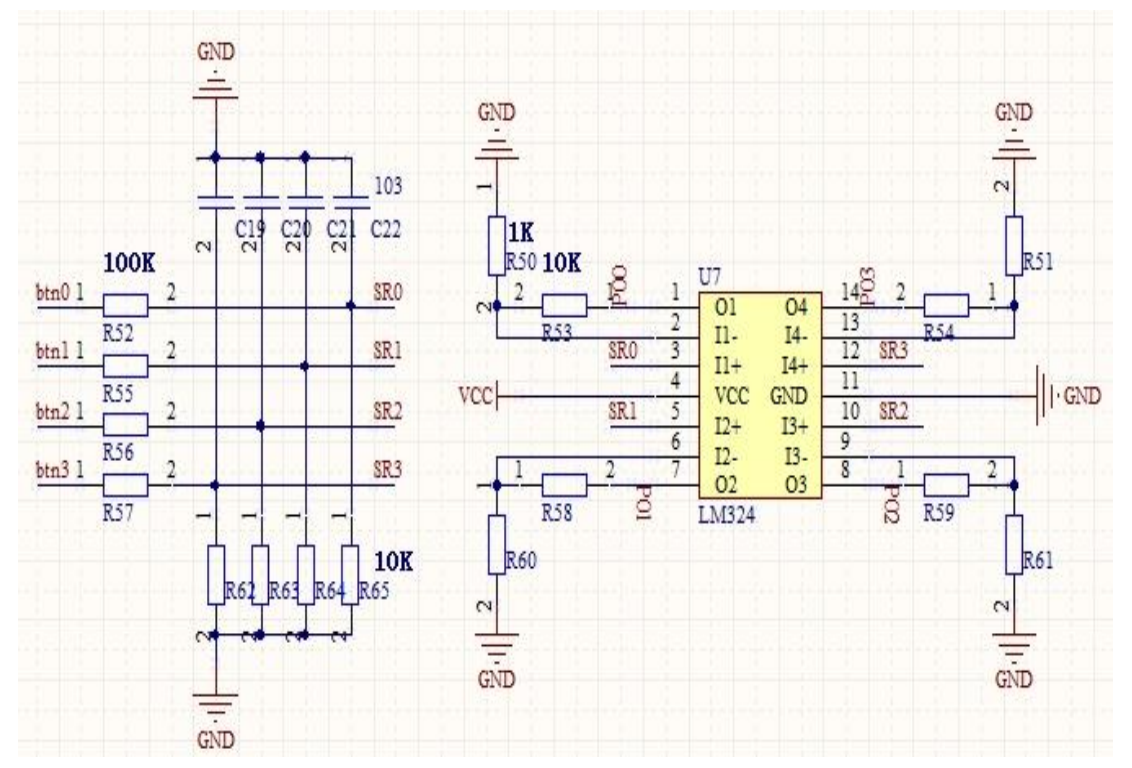

Figure 3. The Acquisition Circuit of Pulse Signal and Switch Signal

2) Circuit of Power Supply Module

Power supply circuit was designed for stepping down vehicle supply voltage of $12 \mathrm{~V}$ to the required working voltage of monitor terminal. Considering the working voltage of MCU chip STC15W4K32S4 was 2.5-5.5V, Bei Dou UM220 module 3.3V, data acquisition module $5 \mathrm{~V}$, and Huawei GTM900-C module 3.8-4.2V, power management chip LM2596-5.0 was introduced to change vehicle supply voltage of $12 \mathrm{~V}$ to $5 \mathrm{~V}$ for power supply of the data acquisition module. Similarly, LM1117-3.3 was applied to transform the voltage of $5 \mathrm{~V}$ to $3.3 \mathrm{~V}$ for the power supplies of the MCU and BDS. In addition, on account of the instantaneous peak current of the GTM900-C module could reach more than $1 \mathrm{~A}$ in the data communication, a separate power management chip LM2596 - ADJ was added, which could reduce the vehicle supply voltage of $12 \mathrm{~V}$ to $4.2 \mathrm{~V}$ and supply power for the GTM900-C module. A standby power supply circuit was designed in case that the monitoring terminal powered down. Once the vehicle supply power was off, the power supply could switch to standby power to maintain the running of monitoring terminal automatically. Figure 4 displays the circuit of power supply module. 


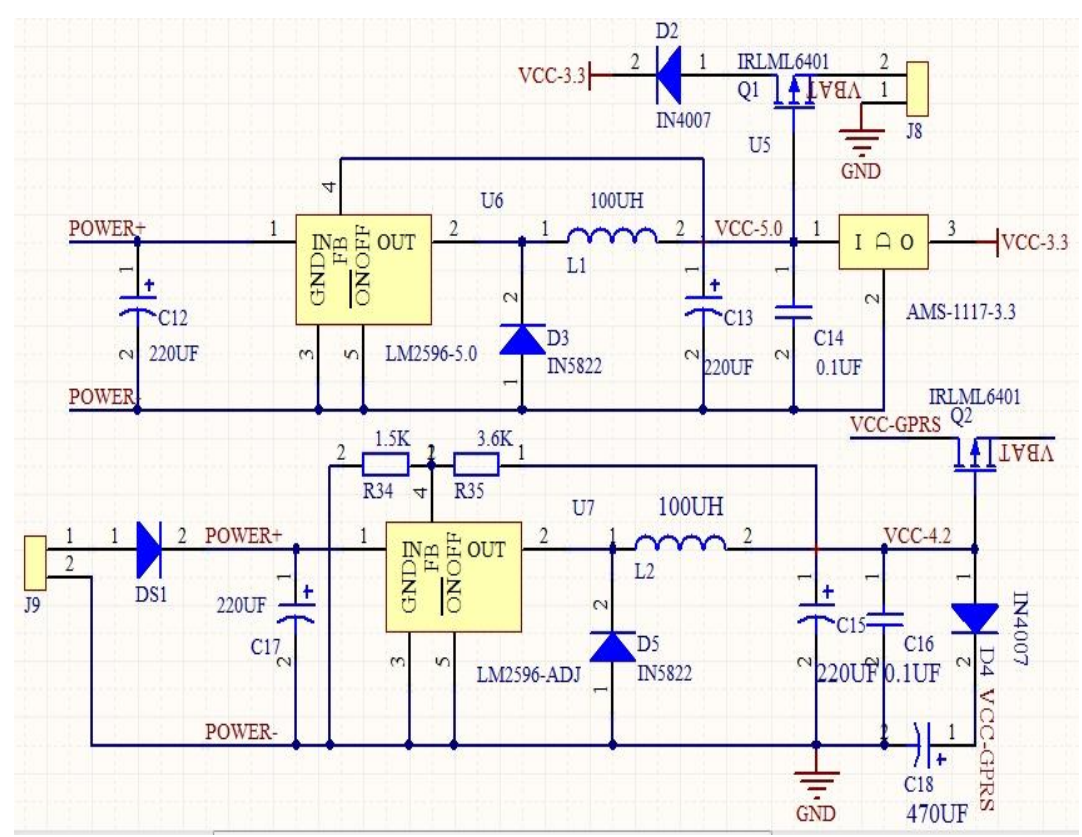

Figure 4. The Circuit of Power Supply Module

\section{3) Positioning and Communication Module}

Bei Dou positioning module UM220 has three UART. UART3 reserved as a special interface for firmware upgrade of the module, UART1 and UART2 were both used as the output interface of data frames in this module. Since the working voltage of UM220 module and MCU module is exactly the same, the UART1 of UM220 module was connected to the UART2 of MCU module directly in this design.

The UART of communication module GTM900-C was attached to the UART3 of MCU module directly. The working voltage of GTM900-C was $4.2 \mathrm{~V}$, which was coordinated with the voltage of MCU module. To solve this problem, RXD interface of UART3 was set to the high impedance state and TXD interface was changed to the push-pull mode in embedded systems of MCU module.

\section{Monitoring Terminal Software Design}

\subsection{Selection of System Platform}

MCU STC15W4K32S4 chip was employed in the monitoring terminal using MCS51 chip as framework for structure. The developing software Keil uVision in MCS51 chip had functions of editing, compiling and simulating. RTX51 Tiny in Keil uVision is a kind of small build-in RTOS with benefits of compact, fast speed, small system overhead and easy to use. The running efficiency of RTX51 Tiny on the 51 single chip microcomputer is better than that of other common RTOS. In view of requirements of multitasking and real-time in this design [8,9], RTX51 Tiny in Keil uVision was chosen as embedded system platform.

\subsection{Division of Program Structure}

In the terminal system, each function module was divided into four different system tasks, including system initialization, GPRS communication, positioning, and vehicle state information. The structure of software system of monitoring terminal is shown in Figure 5 . 


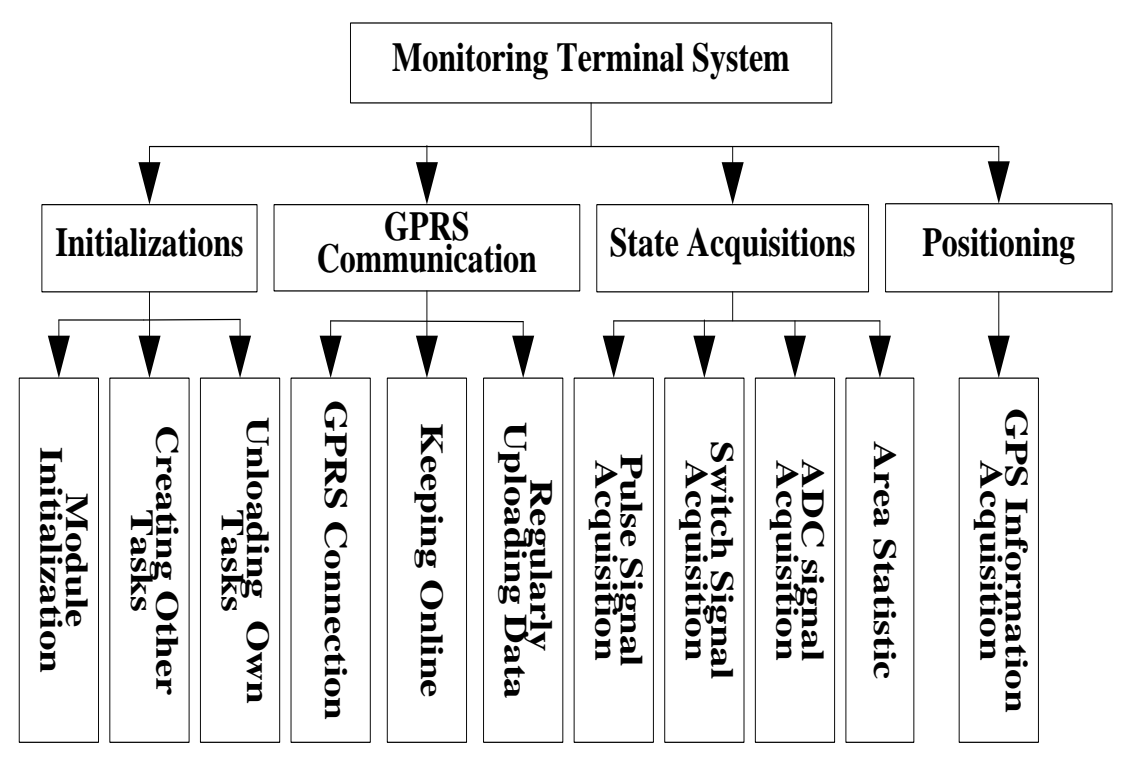

Figure 5. The Structure of Software System in Monitoring Terminal

\section{1) Initialization Tasks}

In RTX51 Tiny real-time system, all tasks must be started with initialization. Timer, UART, ADC function, LCD and GPRS module were firstly initialized after monitoring terminal powered on. Initialization of GPRS module was launched by pulling down its level of PWON pin and the pulling down time was kept not less than $50 \mathrm{~ms}$ before release using MCU port. When MCU UART3 received the string "ready", indicating GPRS module had been booted up successfully. Then the GSM and formats of GPRS communication, such as baud rate and coding format, were established.

2) GPRS Communication

MCU transmitted the corresponding Attention (AT) commands to carry out the operations of the GTM900-C module via UART3. The GPRS linked AT commands of GTM900-C module is shown in Table 1.

Table 1. The GPRS Linked AT Commands

\begin{tabular}{cc}
\hline AT command & Instruction \\
\hline AT\%ETCPIP & Initialization \\
AT\%IOMODE & Data modeling \\
AT\%IPOPEN & Opening one TCP or UDP \\
AT\%IPSEND & Data transmission \\
AT\%IPCLOSE & Cutting off links \\
\hline
\end{tabular}

MCU firstly sent the commands of AT\%ETCPIP and AT\%IOMODE to GTM900-C module, subsequently the transmission of AT\%IPOPEN command of connecting TCP was allowed. After the completion of the module was connected with the monitoring center, MCU would send the packaged data to the monitoring center every 15 seconds using the command of AT\%IPSEND through the GTM900-C module.

3) Vehicle State Information Acquisition

Monitor terminal acquires switch signals including start-stop of tomato harvester, rise-fall of the pick-stage and analog signals of oil temperature and diesel mass. The speed signals of blower and engine were detected by programmable counter array module (CCP/PCA) in the MCU in the way of capturing the pulse widths, and these speed signals were converted to speed values via the rotational speed conversion formula as follows: 


$$
R=60 \frac{N}{n}
$$

Where $\mathrm{R}$ was speed value( $\mathrm{r} / \mathrm{min}$ ), $\mathrm{N}$ was the clock number of CCP/PCA when the time interval was one second, and $\mathrm{n}$ was the cumulative number of clock during two consecutive pulses.

Domestic tomato harvester employed "Mu" as unit of harvesting area, daily used unit of area in China, for pricing. As tomato harvester reached three conditions simultaneously including dropping of harvesting platform, running of chain harrow, and the advancing of whole machine, monitor terminal added up the number of pulses of gearbox sensor and exerted the formula of area algorithm to obtain statistics of area. The formula of area algorithm was:

$$
S=k M \frac{X L}{N} D
$$

Where $\mathrm{k}$ was coefficient of area, $\mathrm{M}$ was number of $\mathrm{Mu}$ corresponding to one square meter $(\mathrm{M}=0.0015), \mathrm{X}$ was the total number of output pulses of gearbox, $\mathrm{L}$ was the distance the vehicle forwarded as the tire rotated one round, $\mathrm{N}$ was number of output pulses of gearbox as the tire rotated one round, and D was the wheel span of tomato harvester.

4) Acquisition and Analysis of BDS Positioning Data

Once the UART2 of MCU received the first character of "\$" and the sixth character of "C", this indicated that the positioning data frames of current string were effective. The position of BDS module was valid as the character was "A", which was after the second comma in the data frames, and the analytic function of BDS, subsequently, extracted information in the data frames including the time after the first comma, the latitude and longitude after the third comma and the date after the ninth comma. If the BDS module could not locate the position of tomato harvester effectively, MCU would ignore positioning information and upload other data to the monitoring center in real time and automatically.

\subsection{Data Communication Protocol Formulation}

Monitoring terminal and monitoring center needed to build a set of communication protocol of the same rules. Monitoring terminal packaged the vehicle state information and positioning data according to the protocol, and uploaded these messages to the monitoring center through GPRS. The monitoring center accomplished the analysis, storage, and display of data, and, meanwhile, realized the judgments of positioning and operating status of tomato harvester after it received the corresponding data frames.

According to the communication protocol, one set of data frame collected and packaged by monitoring terminal was:

(SH0004DD023807A4418.416208633.59120721051404A1005B12242038040107100000 000000160054)

The protocol was mainly composed of the frame header, time, date, location, working status of vehicle motion mechanism, statistics of area and the frame tail. The main packaging program included the following several parts:

\section{1) Time Signals Packaging}

The UTC time "023807" extracted from the data frame by MCU data frame were acquired from the BDS module, were saved in bytes ranging from 10 to 15 , which suggested the point-in-time 2:38:07. Similarly, the UTC date "210514", which suggests the date May 21st, 2014 were written in the bytes ranging from 38 to 43. 
2) Positioning signals packaging

The latitude and longitude information was also extracted from data frames of BDS by MCU. Valid positioning identifier "A" (the invalid positioning identifier was "V") was saved in the 16th byte. For the nine latitude of "4418.4162" and ten longitude characters of "08633.5912", they were saved in bytes ranging from the 17th to 25 th bytes and the 26th to 35th bytes respectively.

3) State signals packaging

The switch signals such as the rise-fall of the pick-stage, start-stop of chain harrow, blower and color sorter, and extend-retract of the uploading conveyor chain of the tomato harvester were written in the bytes ranging from 36 to 43 . The speed values of engine and blower ("1224" and "2038", respectively) were written in the bytes ranging from 44 to 51.The conversion results of the analog signals oil-temperature "0401" and diesel mass " 0710 " were written in the bytes ranging from 52 to 59 . Remember the conversion results should keep a decimal place and then multiply ten. The present harvesting area "0016" and accumulative harvesting area "0054" were saved in 68th to 71st bytes and 72nd to 75th bytes, respectively. The eight bytes from 60th to 67th in the data frame were reserved as the extended collection switch signal.

\section{System Test}

\subsection{Terminal Data Acquisition Test}

Monitoring terminal was installed in the tomato harvester (model of G89/93 MS 40, GUARESI Company, Italy) and tested in the Xinjiang Zhongji red industrial park, Xinjiang Urumqi, China.

The instantaneity, sensitivity and stability of the signals such as vehicle switching state, speeds of blower and engine, gearbox pulses and the oil temperature were tested. The test method of coefficient of work area was: computing the errors between statistical work area obtained from the terminal and the actual work area as the tomato harvester worked 500 meters long, and the experiment was repeated for several times. According to the test results, the error of harvesting area of tomato harvester is less than $1 \%$, when $\mathrm{k}$ was 0.974 .

\subsection{The Monitoring Terminal and Monitoring Center Running Alignment Test}

The instantaneity and reliability of positioning of monitoring terminal and GPRS communication were tested by monitoring center [10]. Forty-three tomato harvesters in red industrial park of Zhongji were employed as the test objects. Monitor terminal uploaded the test data to the monitoring center, and then these data were processed and displayed by the monitoring center software. Figure 6 is the playback of historical track of number C129 car tested. 


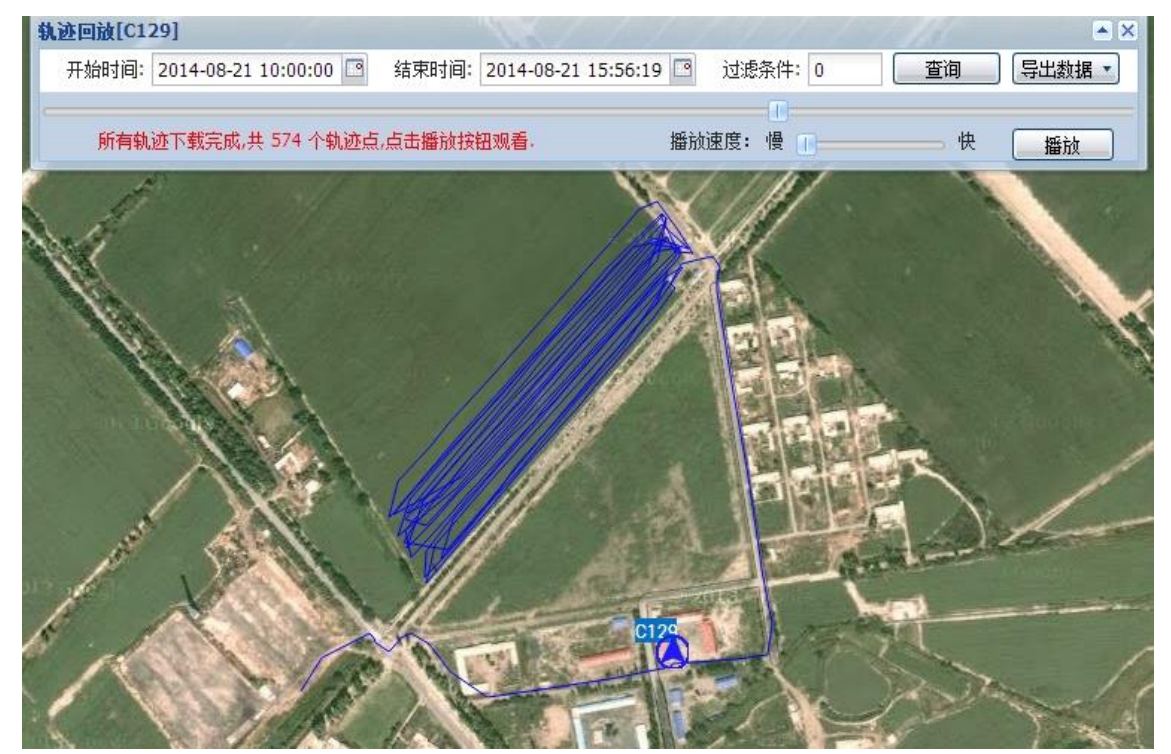

Figure 6. The Playback of Historical Data through Monitoring Center
Software

\section{Conclusion}

By the application of monitoring terminal in tomato harvester in red industrial park of Xinjiang Zhongji tomato product Co.Ltd during tomato harvesting in 2014, it proves that tomato companies can locate the position of tomato harvester rapidly with high accuracy. The current state of tomato harvester could be obtained by using the monitoring center software simultaneously.

The remote monitoring terminal with GPRS communication technologies and BDS localization is one important part of the tomato harvester remote monitoring system. The application of the monitoring terminal could provide reliable technical supports for safety production and scientific scheduling of tomato companies. Furthermore, it is significant to the development of informationization of the tomato harvesting management, and this design could give a reference for the development of intellectualization of other agricultural machineries.

\section{References}

[1] P. Roblin and D. A. Barrow, "Microsystems technology for remote monitoring and control in sustainable agricultural practices", Journal of Environmental Monitoring, vol. 2, no. 5, (2001), pp. 385-92.

[2] J. F. Lu, "A Discussion on an Efficient Virtual Traffic Control System for Un-Trackable City Vehicles", Energy Procedia, vol. 17, (2012), pp. 1261-1267.

[3] "Determination of the Differential Code Bias for Current BDS Satellites", IEEE Journal on Transactions on Geoscience and Remote Sensing, vol. 52, no. 7, (2014), p. 1.

[4] H. S. Kim, D. S. Kang and J. H. Kim, "The BDS statistic and residual test", Stochastic environmental research and risk assessment, vol. 17, no. 1/2, pp. 104-115.

[5] T. M. Yang, W. Xiao, andJ. L. Guo, "Design of Taxi Vehicle Monitoring System Based on GPS/GPRS", 3rd International Conference on Artificial Intelligence and Computational Intelligence, (2011).

[6] Z. C. Huang, D. F. Huang, Z. Xu and Z. G. Xu, "GPS Vehicle Positioning Monitoring System Integrated with CORS and Mobile GIS", Procedia Environmental Sciences, vol.10, (2011), pp. 2498-2504.

[7] S. W. Li, J. J. Tian, Z. F. Yang and F. Y. Qiao, "Research and Implement of Remote Vehicle Monitoring and Early-warning System Based on GPS/GPRS”, Energy Procedia, vol. 11, (2011), pp. 134-140.

[8] G. L. Li, D. Y. Zhang, J. Zeng and S. H. Chen. 


\section{Authors}

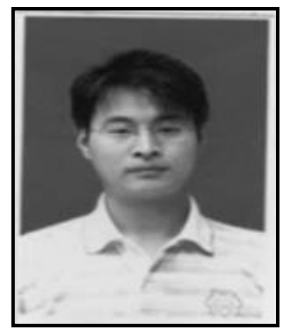

Bin Li, he graduated from Liaoning Project Technology university of Electrical engineering and automation in 2012, now studying in Shihezi University as a master. His scientific interests is intelligent detection and automatic control technology.

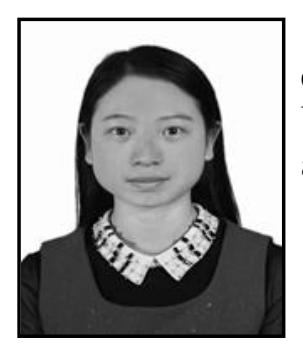

Danyang Li, she graduated from Shihezi University of Electrical engineering and automation in 2013, now studying in Shihezi University as a master. Her scientific interests is intelligent detection and automatic control technology.

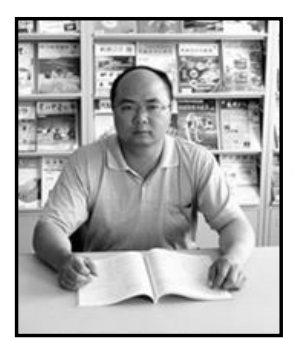

Jiangquan Li, he graduated from Northwest agricultural and forestry of Agricultural machinery manufacturing, postgraduate supervisor in the Department of Automation and Electrical Engineering, Shihezi University. His current position is postgraduate supervisor. He publicated 45 books.His scientific interests is intelligent detection and automatic control technology. 